\title{
8. Studies on Secondary Pathways of the Trigeminal Nerve and Forel's Tegmental Fascicle in Cats
}

\author{
M. KURU \\ National Cancer Institute \\ K. Akagi, R. Kuroda and T. Hayakawa \\ The 2nd Surgical Clinic, Osaka University Medical School
}

The spinal nucleus of the trigeminal nerve and its surrounding tissues were destroyed in 16 cats and the successive degenerations were traced with Marchi method.

Three systems of the secondary pathways of trigeminal nerve were demonstrated, confirming Kuru's finding in human brain stem. The first pathway coincides with Wallenberg's dorsal tract and participates in Forel's tegmental fascicle. The second pathway is identifiable with Spitzer's ventral tegmental fascicle. The third pathway, presumably mediating the pain and temperature sensations from the face, takes the analogous course to that in man (Kuru) and provided with considerable number of fibers. All these three tracts originate in the nucleus of the spinal tract of the trigeminus and terminate in the arcuate nucleus of the thalamus.

Forel's fascicle contains another component of ascending fibers. The related fibers originate in the gray matter column situating dorsomedial to the spinal tract of the trigeminus. The caudal division of this column corresponds to the nucleus juxtasolitarius in man (Kuru) and the rostral division to the gustatory nucleus of Grossmann. This component of Forel's fascicle terminates mainly in the central gray substance of the mesencephalon. Reasons are presented for the view that this tract is concerned with mediation of visceral sensations inclusive of the gustatory and sexual sensations.

\section{Our Surgical Experience of Trigeminal Spinal Tractotomy (Sjöqvist) Procedure Modified}

\author{
Kinjiro Iwata, Akira Asai, Saburo Watanabe, Norihide \\ Nakashima, Masamitsu Nakajima and Koji Nakamura \\ First Surgical Clinic, Nagoya University School of Medicine
}

For the treatment of trigeminal neuralgia or symptomatic facial pain, Gasserian ganglion block or Gasserian posterior rhyzotomy has been accepted as the standard procedure. We do not have any objection on this point; however, we encounter 
cases occasionaly which are not successful because of the use of these standardized procedures or because of regional pathology such as invasion of maxillary cancer. The procedure has to be done elsewhere away from Gasserion ganglion. In these instances, which are not very common, we chose the Sjöqvist procedure in five cases. In this country, the experience with this procedure is rarely found in the literature, but we feel that this procedure is still one of the useful ones in the field of pain surgery. We will report our five cases with special emphasis to the result of the operation and problem of recurrance of pain.

\title{
10. Studies on the Insensible Water Loss in the Neuro-Surgical Patients
}

\author{
Yasuhiro Hirayama, K. Ishizuka, M. Morooka, \\ S. Nomura, K. Ito and S. KiKuchI \\ Juntendo Univ. School of Medicine, Department of 2nd Surgery
}

To estimate the insensible water loss from the surface of the Neuro-surgical patients, authors have utilized a electrohygrometer by measuring the humeridity above the skin surface.

In the preliminary experiments, the water loss from the various parts of the body surface of the normal healthy individuals and its seasonal variation was estimated.

In the clinical appliance, a group of 33 cases with brain tumor showed marked difference on the insensible water loss between its value estimated on the back of the hand of each side, whereas no difference was observed by a normal control group of the healthy individuals and 60 cases with head injury. Such tendency was especially remarkable in cases of brain tumor, such as hypophysial tumor, third ventricle tumor and so on.

In the further investigation in a group of brain tumor, authors studied the relation between the bilateral asymmetry of quantity water loss on the back of the hand and the operation, and found its marked postoperative difference between both sides. 